\title{
Leaps of Faith in the Obesity Debate: a Cautionary Note for Policy-makers
}

LINDA COURTENAY BOTTERILL

Barely a day seems to go by without a media report addressing the 'obesity epidemic' that is apparently afflicting developed countries. Public debate on the topic invariably calls for government action: from information campaigns to educate consumers about food choices and encourage increased physical activity, through to regulation of television advertising of 'junk' food (particularly to children), limiting access to such food in school canteens and taxing fat content in food. Current affairs television regularly carries stories on obesity, as do the print media, and government websites in the United Kingdom, the United States and Australia include advice on healthy weight and the risks of obesity. Calls for government intervention in response to obesity are based on a number of propositions that, on closer inspection, suggest caution by policy-makers before they respond. This article explores the basis for these calls to action, and raises some questions about the uncertainties that they disguise and the leaps of faith that we are being asked to take in promoting government intervention in what we eat and how active we are. The propositions that need closer scrutiny are as follows: first, that we know what is causing weight gain and how to reverse it; second, that there is a proven link between individual weight gain and increased disease risk; and third, that if we do not act now, public health systems across the developed world will collapse. The fourth proposition is that weight loss is risk free and the final proposition is that scientific evidence is objective. This art- icle concludes with a cautionary note for policy-makers. It begins, however, with a note on the measurement of obesity and overweight $^{1}$ and the impact this has on the public debate.

\section{A note on the magnitude of the problem}

From the outset, it should be noted that the obesity debate is subject to hyperbole, much of which can be attributed to the metric that is used to identify the problem. The Body Mass Index (BMI), which is at the basis of calculations of the proportion of the population that is obese, is a very crude measure of health status. An individual's BMI is his or her weight in kilograms divided by his or her height in metres squared $\left(\mathrm{kg} / \mathrm{m}^{2}\right)$. The 'ideal' BMI is generally agreed to be in the range of 18-24.9, with 25-30 being considered overweight and over 30 considered obese. This figure is not adjusted for very fit or muscular individuals, which means that the percentage of the population assessed to be overweight includes extremely fit and healthy individuals whose BMI is high due to the fact that muscle is heavier than fat. On this basis, Brad Pitt and Michael Jordan are overweight, while Russell Crowe and George Clooney are obese. ${ }^{2}$ The alarmist numbers used in the obesity debate are also a result of the conflation of overweight and obesity. This means that an individual of 180 $\mathrm{cm}$, or just under six feet tall, is included in the figures if he or she weighs over 81 $\mathrm{kg}$, or just over 178 pounds. While there is 
undeniably disease risk associated with obesity, it is debatable whether there is increased disease risk associated with mild overweight. Conflating overweight and obesity allows advocates for action to argue that a large percentage of the population is 'overweight or obese' but, of this number, only a very small proportion is actually at significantly increased disease risk.

The inflated numbers being used to describe the problem are related to the use of the word 'epidemic'. Epstein ${ }^{3}$ suggests that "The constant use of the term "epidemic" does more to inflame than inform. Whatever the problems with obesity, it is not a communicable disease, with the fear and pandemonium that real epidemics let loose in their wake.' Nevertheless, some of the language of the literature implies that obesity is indeed 'catching', with researchers suggesting that anti-obesity strategies need to be two-pronged, including both those already overweight/obese and also those who are of 'normal' weight. Medicalisation of obesity allows the issue to be seen as a public policy problem rather than a focus for the private, or domestic, sphere. It is of course arguable whether it makes sense to classify particular body size as a disease in its own right, on the basis of its association with other medical problems.

Putting the numbers to one side, there remain some problems with the obesity debate of which policy-makers should be aware before they respond to exhortations for action.

\section{Proposition 1: The causes of weight gain are known and effective solutions are available}

The first leap of faith being made in the obesity debate is that we know what causes weight gain and that we also know how to make fat people thin. Much of the debate about the subject and the proposed solutions subscribes to the 'energy-in, energy-out' view of weight gain. However, even this apparently uncontroversial understanding hides a number of different interpretations and underpinning values. At its crudest, weight gain is seen as an individual failing. It involves two of the seven deadly sins: sloth and gluttony. If we all ate a little less and exercised a little more, we would not be gaining weight. We are encouraged into this slothful lifestyle by the ready availability of sedentary pastimes such as television viewing and playing computer games-activities that researchers often link with the consumption of high-energy foods. It is worth noting that the diet industry is an important beneficiary of this view. If a particular weight loss programme fails to deliver results, this outcome can be attributed to the client's lack of willpower rather than the shortcomings of the product they have purchased. This tendency to blame the overweight individual for failure can also be found in the scientific literature, 'cheating' or inaccurate reporting of food intake or exercise by trial participants sometimes being implied when research results are mixed.

Alternatives to the sloth-gluttony interpretation see obesity not as a failure of willpower, but as a failure of our genes. There are several variants on this. One is related to the 'energy-in, energy-out' approach, but shifts the blame from the individual to the human gene pool and the mismatch between hunter-gatherer physiology and modern lifestyles. The solution is still related to the energy balance, but the moral judgement about cause has shifted from the individual's failings to the features of modern living. A key problem with this explanation is that the obesity epidemic is generally considered to be about 20 years old and it is considerably longer than that since humans were predominantly huntergatherers. Another version is that some individuals are more prone to weight gain than others due to their genetic 
make-up, and there are scientific studies that support this.

A further explanation is that weight gain is a societal failing. In this interpretation, modern lifestyles create an 'obesogenic $^{\prime 4}$ environment by promoting increased food intake and reduced physical activity. Features of modernity that contribute to the obesogenic environment are escalating car reliance, lack of time, rising use of convenience foods and a move away from structured eating patterns. Central heating has also been identified as a problem, as it 'reduces the need to expend energy for thermoregulation and probably encourages lethargy'. ${ }^{5}$ There are clearly some social agendas at play in the public debate, as working mothers have been identified by more than one study as the cause of childhood obesity due to their reduced role in producing wholesome home-cooked meals and their unavailability to supervise non-sedentary activities by their children. In the US, racial and class dimensions have been identified in the debate. As discussed below, extreme thinness is also unhealthy, but this is not a focus of public concern, suggesting that aesthetics also play a part in the obsession with weight gain. The failure of other events to follow the Madrid fashion show's 2006 decision to ban models with a BMI of less than 18 is an indicator of how thinness is valued, even at unhealthy levels.

Research by scientists working on obesity strategies suggests that there is no clear 'cure' for excess weight. It is generally accepted that diets don't work and that in the long run dieters may end up heavier than if they had accepted their starting weight in the first place. For policy-makers concerned about excessive intervention in private behaviour, or the rise of the 'nanny state', education campaigns are often presented as a good starting point for governments. They are not particularly intrusive and are based on the premise that the consumer is rational and, once informed about risks associated with a particular behaviour, will amend that behaviour. However, the evidence is mixed as to the effectiveness of education campaigns in reducing obesity in the population. There is also concern about the negative impact of such programmes on the target audience-particularly the impact of the weight loss message on overweight children, who are conscious of their 'undesirable' body shape and already likely to suffer low self-esteem. Experience also shows that the weight loss message is taken up most vigorously by young women, including those whose weight falls squarely within the 'normal' BMI range of 18-25 and who may increase their disease risk by losing weight.

Some researchers have moved beyond education campaigns to assess the efficacy of various types of direct intervention programmes to prevent weight gain or address existing overweight or obesity. These have also not had a high success rate and generally end with calls for further research to be undertaken. The difficulties associated with weight loss are recognised in the research, leading to the tendency to focus on childhood obesity and the prevention of weight gain by those who are currently young and thin, rather than to seek to address existing adult overweight and obesity. The focus on children and protection of the innocent and ignorant is a tactic that has been used by nutrition crusaders for decades and is seen in the attacks on 'Big Food', which is deemed by its critics to be so powerful that it limits our capacity to make rational food choices.

For those researchers who see the cause of obesity not in individual behaviour but in the obesogenic environment, other strategies are seen as more effective, but large-scale urban redesign to increase activity and promote major lifestyle changes is arguably an impractical policy option. Further research is needed to establish whether environmental or behavioural change would have a significant impact on obesity.

Leaps of Faith in the Obesity Debate: a Cautionary Note 495 
Another approach that has received some attention is direct intervention in the price of 'problem' food. However, a fat tax raises some difficult policy issues: What would be taxed? How would it be calculated to account for different types of food and means of preparation and nutritional value? Wouldn't it penalise people who eat a lot because they are very fit? There is also a social equity issue as increased food costs impact disproportionately on the poor, who are already limited in many communities in their access to fresh, healthy food options.

In his work on preventive medicine, Rose $^{6}$ argues that once public health instruments move beyond approaches involving individual choice (demand-side measures) and are delivered through supply-side measures-that is, involuntarily-'We should expect a higher level of scientific evidence and popular acceptability' of the measures being considered. Popular debate surrounding overweight and obesity implies that anti-obesity measures would be broadly acceptable; however, the scientific evidence underpinning obesity responses remains sufficiently uncertain that this threshold may not have been reached. Intervention in the food supply, either through taxes or regulation of particular foodstuffs or ingredients, should be considered very carefully.

\section{Proposition 2: There is a link between weight gain and increased disease risk}

In many cases, the debate surrounding obesity proceeds as if the relationship between obesity and disease risk were incontrovertibly established. Bagust et al. state that 'a wide range of epidemiological studies have implicated obesity as a significant predisposing risk factor in a variety of disabling and life-threatening medical conditions'. ${ }^{7}$ It is worth unpacking this statement, as it is typical of the approach that forms the basis for much of the debate. First, the claim relates to obesity and not overweight, so the number at risk is relatively smaller than the 'epidemic' discourse-which, as noted, tends to conflate overweight and obesity as a single problem. Secondly, obesity is a 'predisposing risk factor' - not an established causal factor.

Caution needs to be exercised when discussing disease risk. The problem of obesity is identified on the basis of epidemiological studies, which point to a correlation between overweight and obesity in a population with the occurrence of particular diseases in that population. The public health objective of calls for antiobesity campaigns is to bring down the population's average BMI, thus reducing the population's disease risk. Small changes in individual behaviour can have a significant impact on population averages, so generating these changes should result in the desired outcome. However, this raises the problem of motivating individuals who are at low risk to act in the collective interest. This is known as the prevention paradox: ' $a$ preventive measure that brings large benefits to the community offers little to each participating individual'. ${ }^{8}$ Even for those at 'high risk', it may be irrational to change behaviour. For the rational individual, taking difficult preventive action in the face of immediate temptations in order to ward off a very small personal risk does not make a lot of sense. For any given individual, gaining a few extra pounds may mean very little in terms of the personal risk of disease-particularly if the person neither suffers from high blood pressure nor has a family history of heart disease or diabetes.

From a public policy perspective, the problem of motivating individuals to act against their own interest for the public good suggests that the prevention paradox is a form of collective action problem. The rational consumer may hear the health message but recognise that, at a 
personal level, there is little incentive to change behaviour patterns due to the low levels of absolute risk involved. In his seminal study on the problems of motivating individuals to collective action, Olson suggested that this would only happen in latent groups if there was coercion to participate or if the individual received some private, non-collective benefit from participation. ${ }^{9}$ This suggests the need for policy to consider some form of private benefit to provide a sufficient incentive for individual action to control or reduce weight. Reduced health insurance premiums for individuals with healthy BMIs is one option, as are health fund or tax rebates on gym memberships and weight loss programmes. While these are likely to be costly to implement and administer, Olson's analysis suggests that without some form of inducement to act, it is unlikely that a reduction in population BMI through individual action will be achieved.

\section{Proposition 3: The public health system is under threat as a result of increasing levels of overweight and obesity}

The third leap of faith that policy-makers are urged to accept relates to the major rationale for public policy action on obesity. That is, if the obesity epidemic is not addressed, public health systems will collapse under the pressure of obesity-related illness. This argument provides a justification for intervention in an area of citizens' lives, which may otherwise be seen as beyond government reach in a liberal democracy. A classical liberal interpretation of obesity would surely be that over-eating and low levels of physical activity are private behaviours which, at worst, result in harm to the obese person themselves. Others can attempt to educate, persuade or cajole individuals to change their behaviour but, in the absence of that threshold test of harm to others, there is no role for compulsion. The purported cost impact of obesity on the public health system, however, makes weight a legitimate area for government intervention.

Like other areas of the obesity debate, the science, in this case the economics, is uncertain. Cost-of-illness calculations relating to the impact of overweight and obesity are generally incomplete. They do not, for example, take account of the savings from weight gain, such as the lower rates of osteoporosis among heavier women. Campos ${ }^{10}$ points out that 'in Great Britain, more women die from osteoporosis-related hip fracture than from breast, cervical and uterine cancer combined', so reduced levels of osteoporosis may outweigh the increased risk associated with carrying extra weight. To be meaningful, the costs of obesity-related disorders need to be counterbalanced with savings in these areas in which overweight has a protective effect. To be brutally accurate, calculations should probably also include the savings in nursing home expenses of those who die prematurely of obesity-related illness and do not go on to contract diseases of extreme old age that require high levels of care. Economic cost calculations should also take account of the cost of physiotherapy, surgery and other medical expenses that arise as a result of injuries from increased levels of participation in sport and other physical activities.

\section{Proposition 4: There is no risk in losing weight}

The correlation between health risk and BMI is by no means linear. Researchers have described the relationship between weight and health risk as either ' $U$ 'shaped or ' $J$ '-shaped, both of which indicate that extreme thinness is also risky. A BMI of 25 is not considerably riskier than a BMI of 23 and may in fact be healthier than a BMI of 17. While populations with

Leaps of Faith in the Obesity Debate: a Cautionary Note 497 
lower average BMIs have lower disease risks, weight loss itself is not without risk. The science relating to the health effects of weight loss, particularly rapid weight loss and 'weight cycling', is even more sparse than the evidence related to the health impact of weight gain. Much more important to health outcomes is the level of physical activity.

Weight loss is therefore not a universal good. Addressing weight gain as a public policy problem is qualitatively different from other public health campaigns, such as reducing levels of smoking. In the case of smoking there was no down side to everyone's quitting the habit-but there are potential risks associated with universal uptake of the 'eat less, exercise more' message. There are groups in the community for whom weight loss would actually mean increased disease risk.

The key messages that emerge from the literature on obesity relate to uncertainty, particularly the uncertainty surrounding the science of the causes of overweight and obesity and uncertainty about the efficacy of the available solutions. There are also cautionary voices in the literature about the impact of public health messages on non-target groups in the community, as well as on overweight and obese children. Outlining these uncertainties is not intended to imply that the problem is trivial but, rather, to highlight the difficulties facing policy-makers in framing an appropriate and effective response.

\section{Proposition 5: The scientific evidence is objective}

One of the striking characteristics of the scientific literature on obesity is that it has a tendency to step outside the bounds of the science and present prescriptions for action without considering the question of whether obesity is in fact a problem that is amenable to a public policy re- sponse. Advocates of government action recognise the need to engage in the policy process, but see this in terms of convincing government and the community of a particular solution. One article suggested encouraging individuals to make minor behavioural changes to their energy balance by, for example, walking an additional mile every day, taking a few bites fewer at each meal, or reducing portion sizes. ${ }^{11}$ Putting aside how such a proposal would be implemented or monitored, these are very specific recommendations based on a presumption that intervention is warranted and would be effective. Advocates of the need for action continue the conflation of overweight and obesity, with the risk of overstating the extent of the problem. They also fail to address the U-shaped relationship between weight and health risk. Some even go so far as to argue that

Given the high prevalence of obesity in the United States and the acknowledged risk, it would seem appropriate to provide the public with information regarding this well-established relation and to reserve the discussion of benefit or harm associated with leanness for scientific debate. ${ }^{12}$

No justification for this recommendation is given, but clearly a value judgement has been made about the relative risks of obesity and thinness. This is a crucial point for public policy-makers, who need to design approaches that do not address the health problems of one sector of the community while putting another group at increased risk of disease.

The contemporary focus on obesity has many of the characteristics of a 'moral panic'. Goode and Ben-Yehuda ${ }^{13}$ describe a number of criteria that define a moral panic, among which are:

- a heightened level of concern over the behaviour of a particular group and the impact of that behaviour on the rest of society;

- increased hostility towards those undertaking the behaviour of concern; 
- a consensus within society, or parts of society, as to the reality of the threat caused by this behaviour; and

- the disproportionate nature of the reaction to the threat.

The level of media attention given to the obesity problem in recent years indicates a rise in the level of concern, and this has consequences for overweight and obese people. There is evidence of discrimination against overweight people in employment, medical treatment and government service delivery. The disproportionality criterion would also seem to apply. The tendency to use 'overweight and obesity' as one category in reporting the obesity epidemic is resulting in an overstatement of the number of people at increased disease risk, as discussed above-and there seem to be other moral agendas at play. These agendas are beyond the scope of this article; however, their existence adds a further cautionary note for policy-makers who are being urged to address the obesity epidemic. Careful consideration of the types of policy proposals being promoted and identification of the motives of their advocates would be prudent. It should perhaps also be remembered that there are serious business interests with a stake in the obesity debate-on both sides of the equation. 'Big Food' sells us high-energy foods while the diet industry, which is not insubstantial, and health clubs benefit from our desire to address the consequences of overindulgence.

\section{Conclusion: some cautionary notes}

Unfortunately for public policy-makers who are attempting to reduce the average BMI in developed countries, obesity does not appear particularly responsive to either individual or population-based interventions. Individual strategies suffer from the collective action problem, while population-based approaches can work when there is an element of public control in the mix-for example, control of the water supply to ensure that drinking water is disease free or to provide fluoridation-but are severely limited when it comes to the regulation of private behaviour.

The self-evident nature of the 'fact' that overweight and obesity are the result of sloth and gluttony is largely uncontested in popular discourse; however, policymakers need to be more careful. In spite of the general acceptance of the 'energyin, energy-out' approach, the science of weight gain remains uncertain and our knowledge of the linkage between weight and health outcomes is also limited. Unfortunately for informed public debate, uncertainties and caveats disappear as obesity research results move from academic journals into popular science and public debate. Many of the academic articles call for further research as the results of their studies are inconclusive, but this uncertainty is lost as obesity-related stories make their way on to television current affairs programmes and into women's magazines that are selling the latest diet fad.

A further problem inherent in the antiobesity message is the cultural and social significance of food. Unlike other public health issues such as smoking, excessive alcohol consumption or drug taking, food consumption is both unavoidable and an important part of our cultural and social lives. Food preparation and consumption is part of people's daily routines and the structure of their family lives. The muchmaligned tendency for working families to rely on pre-prepared and takeaway food may result from a rational decision to spend more time with the family than in the kitchen preparing a meal from scratch. These socially determined elements of food consumption and exercise patterns cannot be ignored by policymakers-expert advice that is focused narrowly on 'energy-in, energy-out' and that does not recognise the broader con-

Leaps of Faith in the Obesity Debate: a Cautionary Note 499 
text is unlikely to succeed. Non-nutritional concerns therefore need to be incorporated in public policy debate over food and nutrition.

The public debate is proceeding apace on the basis that obesity is a collective problem, that government must 'do something' and that straightforward solutions are available. The policy debate should be more nuanced and considered. Good public policy in this area needs good science to inform it and this includes good political science. If obesity is a collective problem, then the lessons of Olson's work in this field should be considered-how to motivate individuals to act when the rational option is inaction. The values underpinning the various proposals need to be articulated. In order to proceed, hard questions need to be asked about the motivations underpinning calls for action. The power interests of players in the debate could be identified, and the role of various types of expertise in the obesity debate is worthy of discussion. In all of these areas, political scientists and public policy specialists can make a contribution. A good starting point would be to redress the use of misleading statistics about the extent of the problem and begin a rational discussion about what government realistically can be expected to do in influencing private behaviour. Moral panics and hyperbole add more heat than light and do not contribute to sensible policy debate.

\section{Notes}

1 Although 'overweight' is used as an adjective in general usage, it is employed as a noun in the obesity literature.
2 Paul Campos, The Obesity Myth: Why America's Obsession with Weight is Hazardous to Your Health, New York, Gotham Books, 2004, p. xxiii.

3 Richard A. Epstein, 'What (not) to do about obesity: a moderate Aristotelian answer', Georgetown Law Journal, vol. 93, 2005, p. 1367.

4 Garry Egger and Boyd Swinburn, 'An "ecological" approach to the obesity pandemic', BMJ, vol. 315, 1997, pp. 477-80.

5 Andrew M. Prentice and Susan A. Jebb, 'Obesity in Britain: gluttony or sloth?' BMJ, vol. 311, 1995, pp. 437-9.

6 Geoffrey Rose, The Strategy of Preventive Medicine, Oxford, Oxford University Press, 1992, p. 114.

7 Adrian Bagust, B. Lynne Roberts, Alan R. Haycox and S. Barrow, 'The additional cost of obesity to the health service and the potential for resource savings from effective interventions', European Journal of Public Health, vol. 9, 1999, p. 259.

8 Rose, The Strategy of Preventive Medicine, p. 12.

9 Mancur Olson, The Logic of Collective Action: Public Goods and the Theory of Groups, Cambridge, Massachusetts, Harvard University Press, 1965, p. 134.

10 Paul Campos, 'The big fat con story', The Guardian, 24 April 2004.

11 James O. Hill, Holly R. Wyatt, George W. Reed and John C. Peters, 'Obesity and the environment: Where do we go from here?' Science, vol. 299, 2003, p. 855.

12 Ramón A. Durazo-Arvizu, Daniel L. McGee, Richard S. Cooper, Youlian Liao and Amy Luke, 'Mortality and optimal body mass index in a sample of the US population', American Journal of Epidemiology, vol. 147, 1998, p. 748.

13 Erich Goode and Nachman Ben-Yehuda, 'Moral panics: culture, politics and social construction', Annual Review of Sociology, vol. 20, 1994, pp. 149-74. 